\title{
Grasping Pattern Recognition and Grasping Force Estimation For Prosthetic Hands
}

\author{
Bing-Ke Zhang ${ }^{1}$, Guo-Liang Zhong ${ }^{1,2, \text { a }}$ and Hua Deng ${ }^{1,2}$ \\ 1 School of Mechanical and Electrical Engineering, Central South University 410083, Changsha, Hunan, China \\ 2 State Key Laboratory of High-Performance Complex Manufacturing, Central South University 410083, Changsha, Hunan, China \\ a Corresponding author: zhonggl2010@163.com
}

\begin{abstract}
Human's movement can be decoded by surface electromyography (EMG), and the prosthetic hand can be controlled freely through EMG signal. This paper proposes a grasping pattern and force synchronized decoding method for prosthetic hands. Considering pattern recognition and force estimation simultaneously, this paper analyzes whether different muscle contraction levels affect pattern recognition and whether different grasping modes have impact on force estimation, then proposes two schemes to complete EMG simultaneously decoding. Experiments compare the accuracy of the two methods. The results show that there is no much difference between two methods in force estimation, the former's accuracy of pattern recognition is a little higher than the latter.
\end{abstract}

\section{Introduction}

In application, the prosthetic hand should be emphasized to the controllable grasping pattern and controllable force $[1,2]$. In the prosthetic hand motion, not only need to recognize hand posture via the electromyographic (EMG) signal, but also need to analyze what should be the size of the grasping force .In actual work, pattern recognition and decoding should be done at the same time. Therefore, it is important to decode the EMG signal combined with pattern recognition and grasping force estimation.

Over the past decades, in order to improve grasping stability performance, synchronized decoding has been widely used in grasping force control and other aspects [3-5]. By imitating the method of grasping objects used by humans, it is possible to grasp any objects that the weight and friction coefficient are unknown [6-8]. A part of the researchers focus on the grasping used by human hands, try to find the grasping pattern recognition method and obtain the optimum value of grasping force. Wang et al. [9] proposed a method for controlling the grasping force by using finite element analysis based on human hand grasping when the weight of the grasped object is unknown. Besides, a lot of work has been done to estimate parameters by taking advantage of the EMG signal as it can be used to adjust the grasping force to the desired value when the prosthetic hand subjects to external disturbances [10].

In order to realize the stability of the prosthetic hand grasping, EMG signal need to decode for grasping mode and grasping force separately, that is, in the process of grasping, the prosthetic hand is not only able to correctly identify EMG signal of the grasping pattern and accurately estimate the size of the grasping force. Such as grasping an egg, excessive force may make eggs break, if the force is too small it will fall and broken. Therefore, an appropriate grasping force is a key factor to achieve excellent grasping performance. However, the existing studies almost focus on the pattern recognition for the prosthetic hand [11], few of them study the grasping pattern and grasping force synchronized decoding for prosthetic hands. This paper is aim to propose a method of grasping pattern and grasping force synchronized decoding for prosthetic hands.

\section{Feature selection of grasping force estimation based on the EMG signal}

In this paper, we choose the following characteristics that can represent the muscle contraction state in time-domain.

1)Mean Absolute Value (MAV). The mathematical formula can be defined as

$$
M A V=\frac{1}{n} \sum_{k=1}^{n}|u(k)|
$$

2)Integrated EMG (IEMG). The mathematical formula can be defined as

$$
I E M G=\sum_{k=1}^{N}|u(k)|
$$

3)Wave Length (WL). The mathematical formula can be defined as 


$$
W L=\sum_{k=1}^{N-1}|u(k+1)-u(k)|
$$

4)V-order,(vOrder). The mathematical formula can be defined as

$$
v \operatorname{Order}=\sqrt[v]{\frac{1}{N} \sum_{k=1}^{N}|u(k)|^{v}}
$$

5)Willison Amplitude (WAMP). The mathematical formula can be defined as



(a) The curves of actual value and MAV value



(c) The curves of actual value and WL value

$$
\begin{aligned}
& \text { WAMP }=\sum_{k=1}^{N-1}[f(|u(k)-u(k+1)|)], \\
& f(k)=\left\{\begin{array}{lc}
1 & |u(k)-u(k+1)|>\varepsilon \\
0 & \text { else }
\end{array}\right.
\end{aligned}
$$

6)Log Detector (log). The mathematical formula can be defined as

$$
\log D=e^{\frac{1}{N} \sum_{k=1}^{N} \log (u(k))}
$$



(b) The curves of actual value and IEMG value

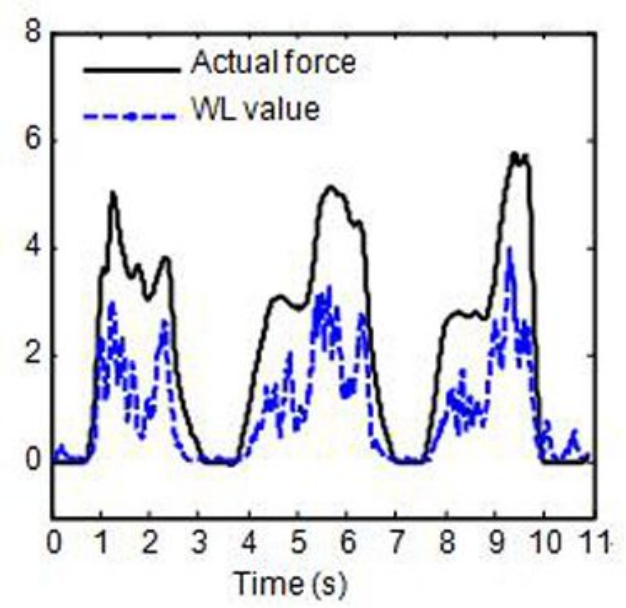

(d) The curves of actual value and vOrder value 




(e) The curves of actual value and WAMP value

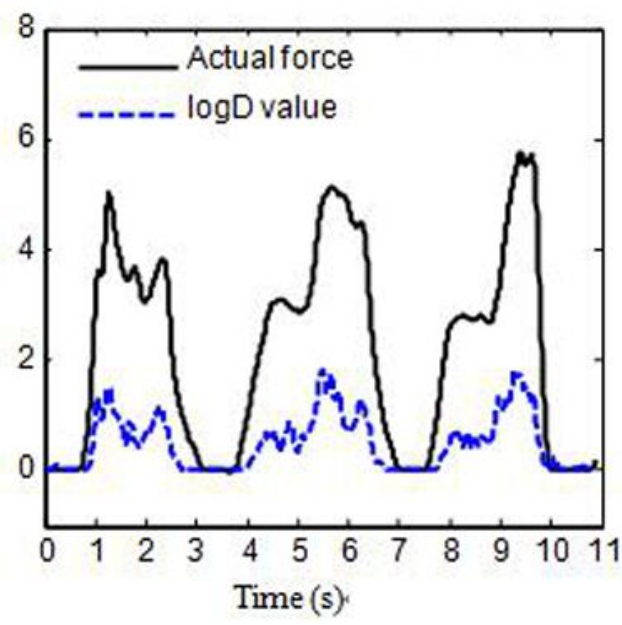

(f) The curves of actual value and $\log \mathrm{D}$ value

Figure 1. The contrast between the Different Eigenvalues and Output Force.



(a) Cylindrical grasping



(c) Finger pinching

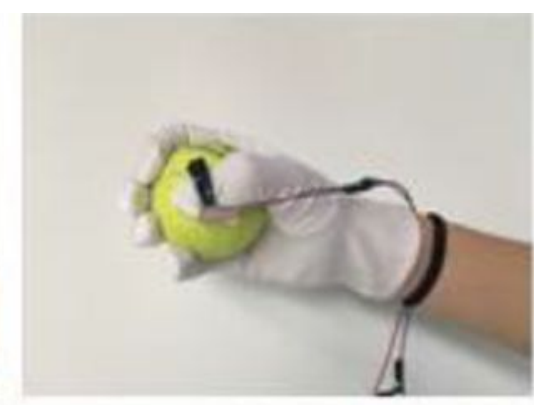

(b) Spherical grasping

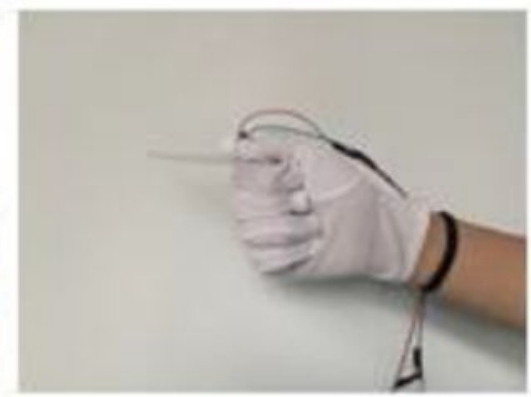

(d) Side pinching

Figure 2. Four Grasping Patterns. 


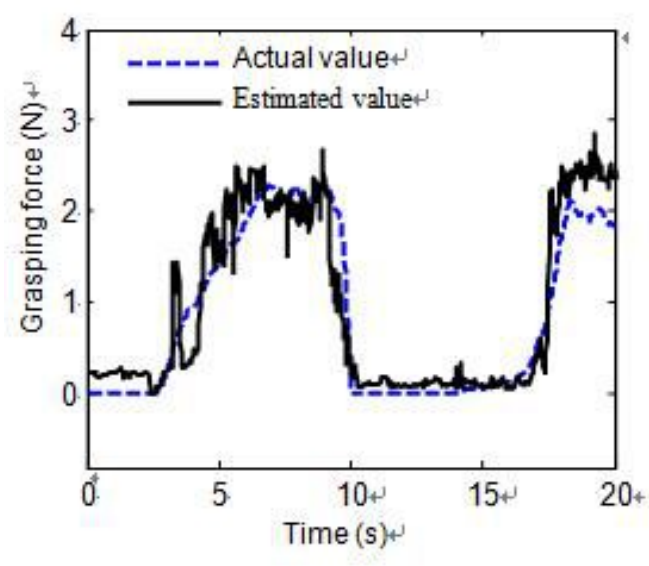

(a) Finger pinching

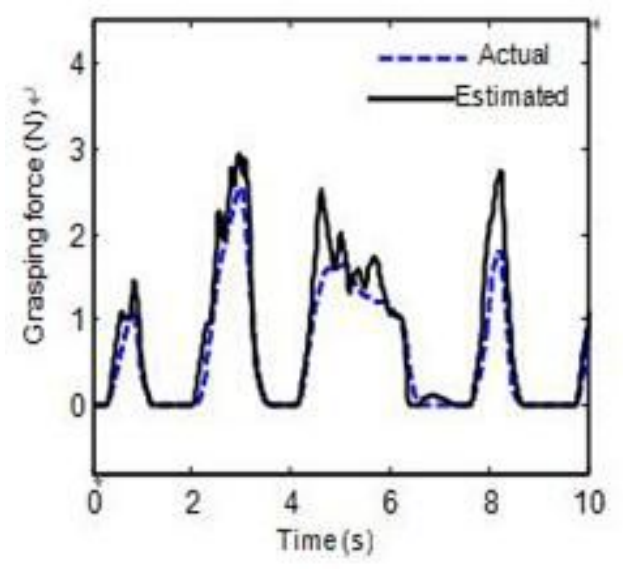

(c) Cylindrical grasping



(b) Side pinching

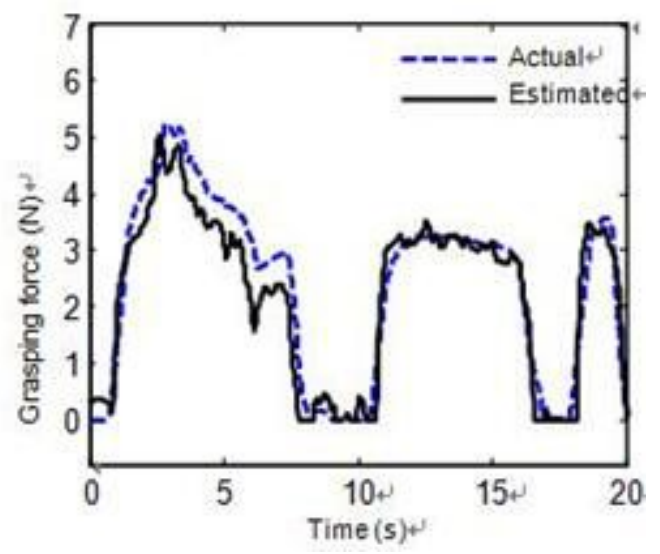

(d) Spherical grasping

Figure 3. Actual Value Estimated Value of Grasping Force Based on Bpnn Method.

Figure 1 shows the comparison between the eigenvalues of the input signal and output force, the EMG signal from the participant who uses side pinched posture for grasping, and the acquisition time for $10 \mathrm{~s}$. The IEMG and WAMP characteristic value is in the range of 0-500. In order to compare the relationship between the characteristic value and the output value, Figure 1 is drew after the eigenvalues of IEMG and WAMP divided by 100 , to make the two eigenvalues and the output force in an order of magnitude.

From Figure 1, we can see that the change trends of six kinds of characteristic value on the output force are the same. Because the EMG signal is muscle cells potential under the stimulation of nerve excitement action, the EMG signal and output force are not perfectly synchronized but has certain hysteresis theoretically. But in terms of EMG signal and the relation between grasping force, and EMG signal response is its excited state and the movement trend, not completely linear or polynomial relationship. Therefore, the regression analysis is needed.

\section{Experimental results}

\subsection{Grasping force estimation}

In order to measure the grasping force easily, this paper first discusses the relationship between grasping force and EMG signal under the four grasping patterns, i.e., the cylindrical grasping, spherical grasping, finger pinching, and side pinching, as shown in Figure 2. In the experiments, experimenter is wearing a FSR gloves in different grasping patterns, and the size of the grasping force is gradually changed in $15 \mathrm{~s}$. After repeating 10 times, participants take 5 minutes to reduce muscle contraction effect on EMG signal.

Compared with the original measurements of smooth, there are different levels of fluctuations in two kinds of predict methods of predictive curves. Correspond to the input signal to a specific value when we make regression analysis. For the multi-channel input, each channel between numerical changes will affect the output signal, and the continuous input signal does not guarantee the continuity of the output value. Because the finger griping force is usually within the range 0 to $10 \mathrm{~N}$, small fluctuation can not affect the trend of predictive value. In practice, it also can grasp the object stably through the 
feedback information and reflex control. Therefore, in

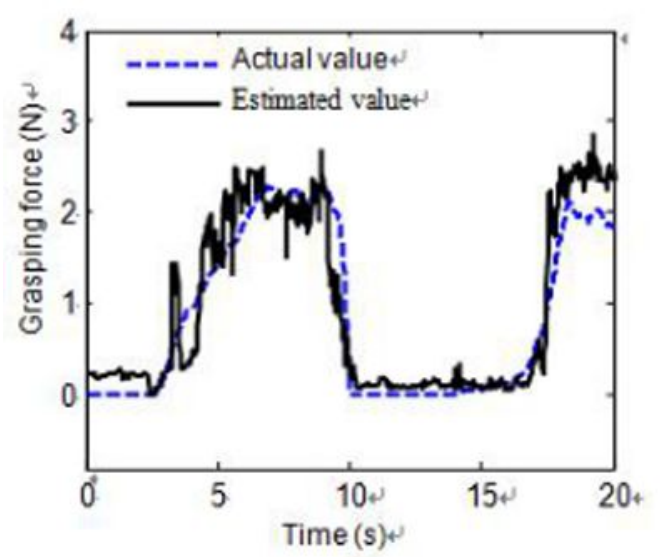

(a) Finger pinching

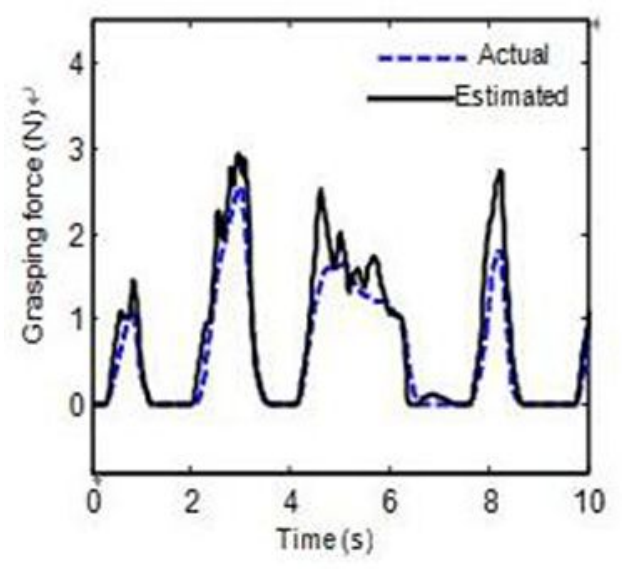

(c) Cylindrical grasping this case, if the predictive accuracy is within $20 \%$, it can realize the force control of prosthetic hand.



(b) Side pinching

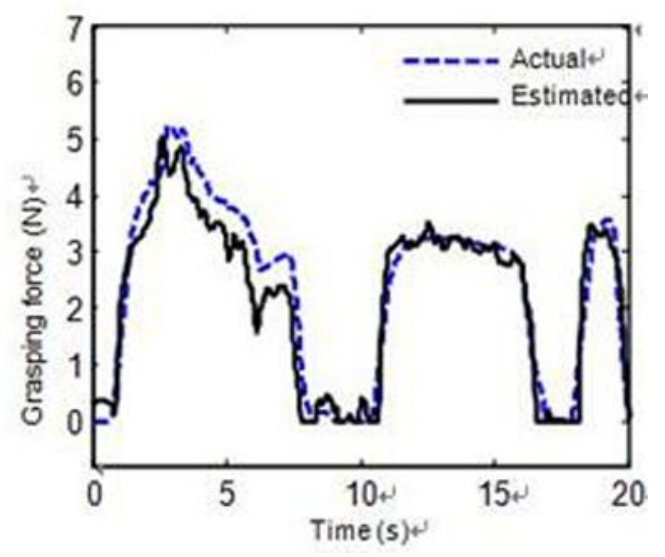

(d) Spherical grasping

Figure 4. Actual Value Estimated Value of Grasping Force Based on Svm Method.



(a) With small force 


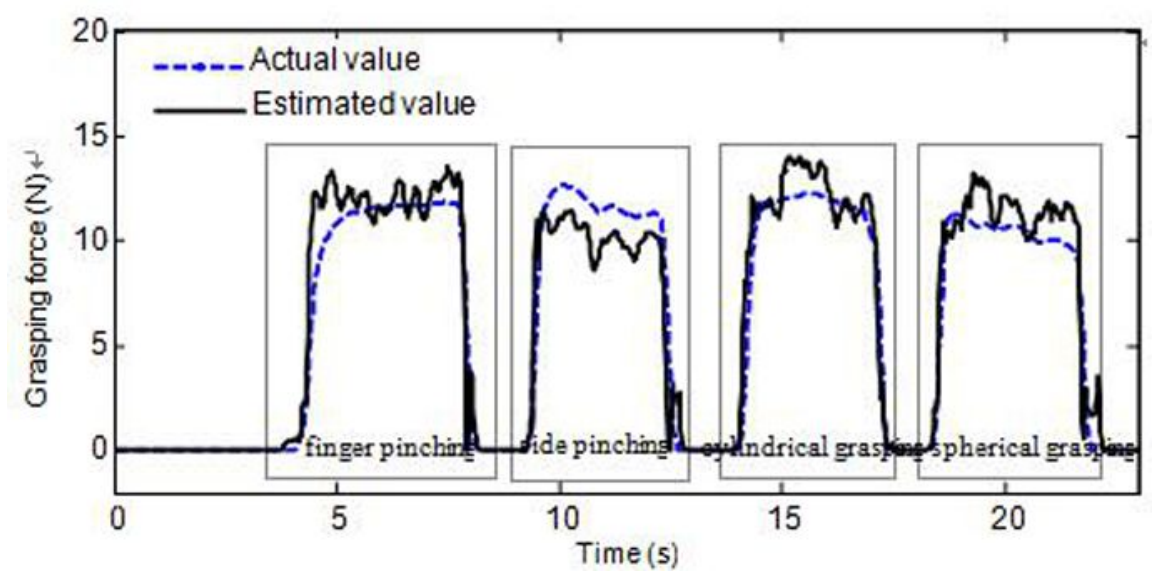

(b) With big force

Figure 5. the Actual Force and Estimated Force under Different Grasping Pattern for Grasping with Small and Big Force.

From Figures 3 and 4, we can see that all three methods can predict the change trend of grasping force, though the error size is slightly different under the correlation of different grasping mode. From the perspective of the evaluation index of MSE and SCC, MSE value becomes minimum while the SCC value becomes largest under the neural network method. That is to say, in three methods, the neural network prediction has the optimal comprehensive performance with minimum error. Under off-line analysis we find that, in the learning process the fitting degree of SVM training data is high, the SCC value is usually over $97 \%$ and MSE value is within $0.1 \mathrm{~N}$. While in the testing, prediction data of SCC value decreases rapidly. The reasons are as follows: on the one hand, some error is existed in model, on the other hand, experience method are used to determine parameters may not be the optimal solution. Therefore, the prediction precision is lower than the SVM method.

\subsection{Synchronized decoding}

Figure 5 draws curves of the actual force and estimated force under different grasping pattern for grasping with small and large force. Due to the small grasping force, the EMG signal is relatively weak, so the precision of pattern recognition is the worst. By correlation coefficient and the MSE can be seen that when we use large grasping force to grasp the object the error is big reached about $20 \%$. The reasons are as follows: on the one hand, due to the different grasp pattern by using the same neural network analysis, the error is bigger, on the other hand, the switch between different modes of action on the recognition accuracy.

Through these experimental results it can be seen that if we first recognize the grasping pattern and then estimate the griping force, precision of the grasping force estimation is higher. However, because the result of pattern recognition are greatly influenced by the degree of muscle contraction, the accuracy of the pattern recognition is low, which affect the invoking neural network. If we first obtain the interval of desired grasping force and then process pattern recognition, the pattern recognition accuracy is improved while precision of the grasping force estimation is reduced. Under this approach, since the initial griping force estimation is in a wide range of interval, we often can accurately determine grasping force interval to choose the right training data for pattern recognition.

\section{Conclusions}

This work studied the influence of different grasping patterns to the grasping force estimation. Experiments showed that the change of grasping pattern would lower the estimation precision of grasping force. In addition, the change of the degree of muscle contraction reduces the pattern recognition accuracy. This paper proposed two kinds of synchronized decoding method of the pattern recognition and grasping force focusing on the above reasons. Namely, one is invoking the corresponding pattern recognition model through the decoding of grasping force, and the other is invoking the corresponding neural network model through the decoding of grasping pattern. Experimental results show that the estimation precision for the two methods is not different, but the former pattern recognition accuracy is higher than the latter. Therefore, synchronized decoding method that invokes the corresponding pattern recognition model through the decoding of grasping force is the better one.

\section{Acknowledgement}

This work was supported by the "973" Program under Grant No. 2011 CB013302.

\section{References}

1. R. Boostani, M. H. Moradi, Evaluation of the forearm EMG signal features for the control of a prosthetic hand, Physiological Measurement, 24(2), 2003: 309-319 
2. M. A. Oskoei, H. Hu, Myoelectric control systemsA survey, Biomed. Signal Proc. Control, 2(4), 2007: 275-294

3. B. Hudgins, P. Parker, R. N. Scott, A new strategy for multifunction myoelectric control, IEEE Trans. Biomed. Eng., 40(1), 1993: 82-94

4. Z. Kermani, M. Wheeler, B. C. Badie, R. M. Hashemi, EMG feature evaluation for movement control of upper extremity prostheses, IEEE Trans. Rehabilit. Eng., 3(4), 1995: 324-333

5. M. Lei, Z. Wang, Z. Feng. Detecting nonlinearity of action surface EMG signal, Phys. Lett. A, 290 (5) 2001: 297-303

6. M. A. Oskoei, H. Hu, Support vector machine based classification scheme for myoelectric control applied to upper limb, IEEE Trans. Biomed. Eng., 55 (8), 2008: 1956-1965

7. D. Farina, R. Merletti, Comparison of algorithms for estimation of EMG variables during voluntary isometric contractions, J. Electromyography \& Kinesiology, 10(5), 2000: 337-349

8. A. Phinyomark Application of wavelet analysis in EMG feature extraction for pattern classificatio. Measurement Science Review, 11, 2011: 45-52

9. G. Wang, Z. Yan, X. Hu, H. Xie, Z. Wang, Classification of surface EMG signals using harmonic wavelet packet transform, Institute of physics publishing physiological measurement, 27, 2006:1255-1267

10. A.Starzacher, B. Rinner, K. N. N. Evaluating, LDA and QDA classification for embedded online feature fusion, International Conference on Sensor Networks and Information Processing, Dec. 2008: 85-90

11. P. Kaufmann, K. Englehart, M. Platzner, Fluctuating EMG signals: investigating long-term effects of pattern matching algorithms, Annual International Conference of the IEEE: Engineering in Medicine and Biology Society (EMBC), Aug. 2010: 63576360 